\title{
Authors response for "Turning chemistry into information for heterogeneous catalysis"
}

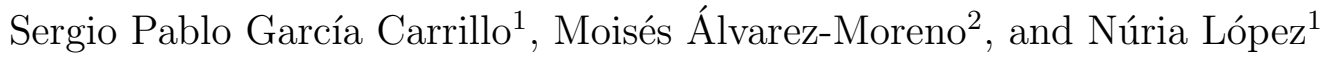 \\ ${ }^{1}$ The Barcelona Institute of Science and Technology, BIST, Institute of Chemical Research \\ of Catalonia, ICIQ, Av. Països Catalans 16, 43007 Tarragona, Catalonia, Spain \\ ${ }^{2}$ Institute of Chemical Research of Catalonia, ICIQ, Av. Països Catalans 16, 43007 \\ Tarragona, Catalonia, Spain, Department of Physical and Inorganic Chemistry, Universitat \\ Rovira i Virgili, C/Marcel-lí Domingo s/n, 43007 Tarragona, Catalonia, Spain
}

June 11, 2020

Open Peer-Reviewer Details for this article are openly available here:

* $\quad$ Peer-Reviewer Report \#1 DOI: 10.22541/au.159182659.98933679

* $\quad$ Peer-Reviewer Report \#2 DOI: 10.22541/au.159182658.82141376

* $\quad$ Editor's Comment DOI: 10.22541/au.159182678.86670339

\section{Hosted file}

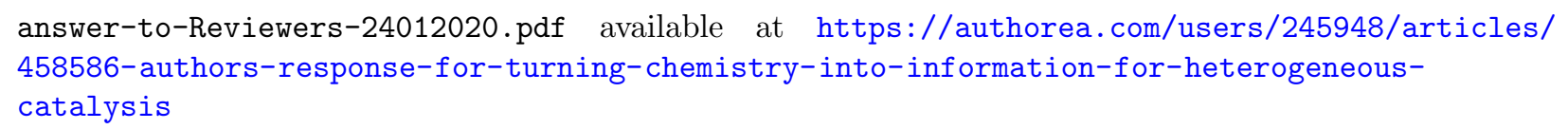

Hosted file

responses_editor.pdf available at https://authorea.com/users/245948/articles/458586-authorsresponse-for-turning-chemistry-into-information-for-heterogeneous-catalysis 